Biofuel Research Journal 18 (2018) 800-805
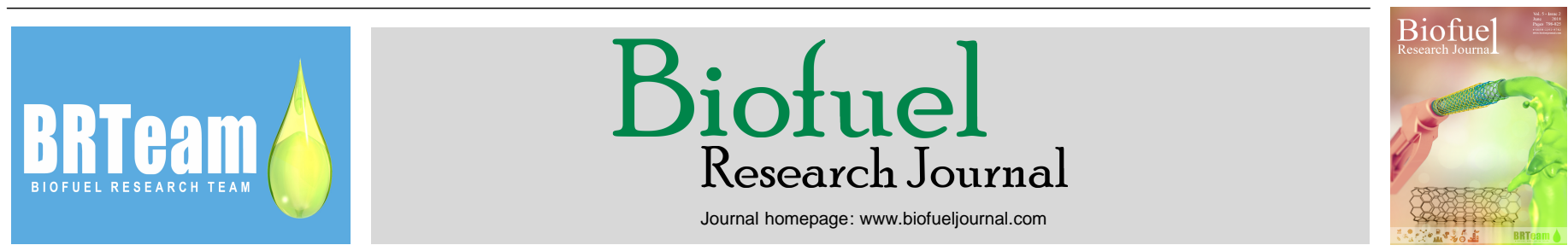

Original Research Paper

\title{
Lipid accumulation from glucose and xylose in an engineered, naturally oleaginous strain of Saccharomyces cerevisiae
}

Eric P. Knoshaug*, Stefanie Van Wychen, Arjun Singh, Min Zhang

National Bioenergy Center, National Renewable Energy Laboratory, Golden, CO, USA, 8041.

\section{HIGHLIGHTS}

$>$ Discovered a strain of Saccharomyces cerevisiae that is naturally oleaginous.

$>$ The engineered strain can utilize the biomass-

derived sugars glucose and xylose to concurrently accumulate lipids and produce ethanol.

$>$ Enables efficient use of biomass-derived sugars for biofuels production.

\section{GRAPHICAL ABSTRACT}

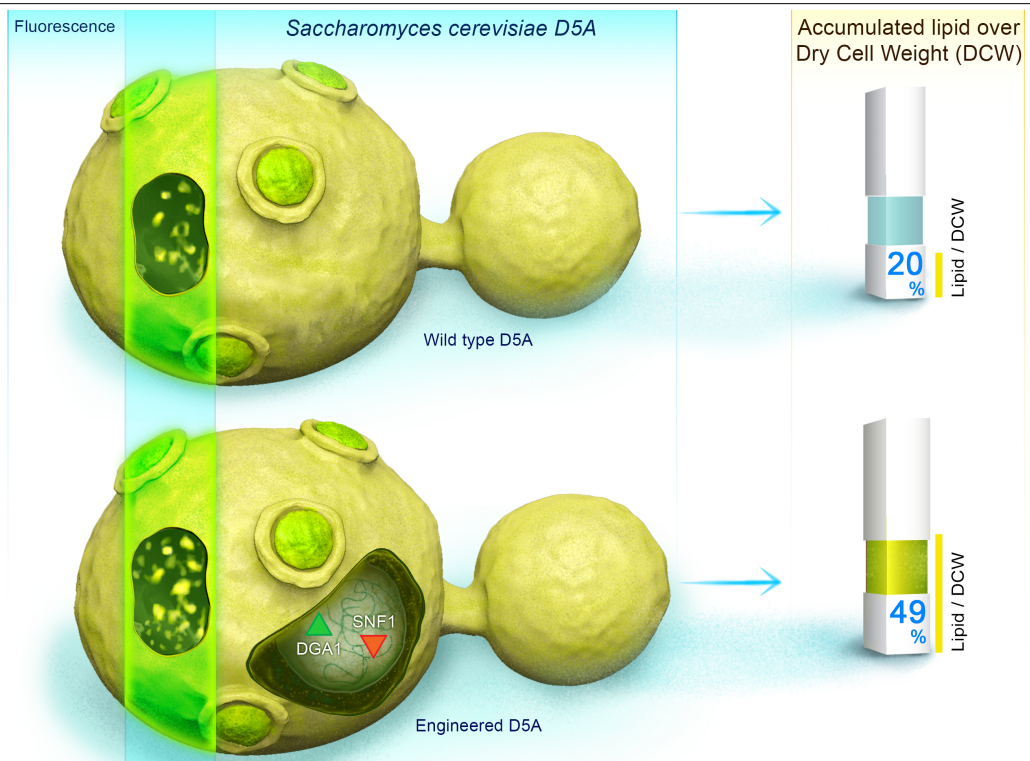

\section{ARTICLE INFO}

\section{Article history:}

Received 17 February 2018

Received in revised form 29 March 2018

Accepted 29 March 2018

Available online 1 June 2018

\section{Keywords:}

Saccharomyces cerevisiae

Xylose

Lipid

Biofuels

Ethanol

Genetic engineering

\begin{abstract}
Saccharomyces cerevisiae, a well-known industrial yeast for alcoholic fermentation, is not historically known to accumulate lipids. Four S. cerevisiae strains used in industrial applications were screened for their ability to accumulate neutral lipids. Only one, D5A, was found to accumulate up to $20 \%$ dry cell weight (dcw) lipids. This strain was further engineered by knocking out ADP-activated serine/threonine kinase (SNF1) which increased lipid accumulation to $35 \%$ dcw lipids. In addition, we engineered D5A to utilize xylose and found that D5A accumulates up to 37\% dcw lipids from xylose as the sole carbon source. Further we over-expressed different diacylglycerol acyltransferase (DGAl) genes and boosted lipid accumulation to $50 \%$. Fatty acid speciation showed that $94 \%$ of the extracted lipids consisted of 5 fatty acid species, C16:0 (palmitic), C16:1n7 (palmitoleic), $\mathrm{C} 18: 0$ (stearic), C18:1n7 (vaccenic), and C18:1n9 (oleic), while the relative distributions changed depending on growth conditions. In addition, this strain accumulated lipids concurrently with ethanol production.
\end{abstract}

* Corresponding author at: Tel.: +1 3033847779

E-mail address: eric.knoshaug@nrel.gov

Please cite this article as: Knoshaug E.P., Van Wychen S., Singh A., Zhang M. Lipid accumulation from glucose and xylose in an engineered, naturally oleaginous strain of Saccharomyces cerevisiae. Biofuel Research Journal 18 (2018) 800-805. DOI: 10.18331/BRJ2018.5.2.3 


\section{Introduction}

Saccharomyces cerevisiae is well known for robust ethanolic fermentation of various pre-treated lignocellulosic feedstocks for renewable fuel production. The two principle monomeric sugars released during pretreatment and enzymatic saccharification of these feedstocks are glucose and xylose. The $S$. cerevisiae strain D5A has previously been used to ferment pre-treated switchgrass, rice straw, distiller's grains, and lodgepole pine feedstocks (Bailey et al., 1982; Geddes et al., 2011) and was found to be tolerant to hydrolysate products present in pre-treated hardwoods and to $1 \%(\mathrm{v} / \mathrm{v})$ butanol (Ranatunga et al., 1997; Knoshaug and Zhang, 2009). However, D5A, like all wild type $S$. cerevisiae strains, is unable to utilize xylose as a carbon source. Through the expression of the bacterial xylose isomerase (Karhumaa et al., 2006; Brat et al., 2009; de Figueiredo Vilela et al., 2013) or fungal xylose reductase and xylitol dehydrogenase pathways (Kotter et al., 1990; Bettiga et al., 2009; Bera et al., 2010), S. cerevisiae has been engineered to utilize xylose to produce ethanol.

The classical definition of an oleaginous yeast is one that accumulates greater than $20 \%$ dry cell weight (dcw) as lipids. S. cerevisiae is not known as being oleaginous and typically only accumulates $10-15 \%$ dcw lipids (Turcotte and Kosaric, 1989; Ratledge and Wynn, 2002; Kamisaka et al., 2007; Meng et al., 2009; Runguphan and Keasling, 2014; Greer et al., 2015) whereas oleaginous yeasts typically accumulate $25 \%$ to greater than $60 \%$ dcw lipids (Ageitos et al., 2011). Recently engineering efforts to increase lipid content have been made in oleaginous yeasts and one target has been the protein kinase SNF1 gene. In S. cerevisiae, SNF1 encodes an ADP-activated serine/threonine kinase (Mayer et al., 2011) that, in addition to its role in regulating carbon homeostasis and general stress responses, regulates genes involved in lipid synthesis and nitrogen metabolism (Usaite et al., 2009; Chumnanpuen et al., 2012) and has been shown to regulate the first committed step of fatty acid synthesis by directly phosphorylating and thus inactivating acetyl-CoA carboxylase (Mitchelhill et al., 1994; Woods et al., 1994). Snf1 ${ }^{-}$mutants have also been shown to be defective in glycogen synthesis (Thompson-Jaeger et al., 1991; Hardie et al., 1998) and $\beta$-oxidation of lipids (Zaman et al., 2008; Usaite et al., 2009), and have shown up-regulation of genes involved in lipid biosynthesis particularly those leading to an increase in the acetyl-CoA pool and the downstream fatty acid synthases and glycerol-3-phosphate dehydrogenase that work in concert to turn that pool into neutral lipids (Seip et al., 2013). In addition, a SNF1 knock-out in the oleaginous yeast Yarrowia lipolytica showed increased fatty acid accumulation over that of wild type in both nitrogen replete and deplete conditions (Seip et al., 2013).

Previous engineering efforts in S. cerevisiae showed a promising increase in lipid content by over-expressing a type 1 plant DGA (Greer et al., 2015). Total lipid production from glycerol was less than $12 \% \mathrm{dcw}$ but represented nearly a doubling over empty vector control (Yu et al., 2013), and up to 17\% dew lipids, a four-fold increase by over-expression of $F A S 1, F A S 2$, and $A C C 1$ with small amounts (up to $4 \mathrm{~g} / \mathrm{L}$ ) of ethanol being produced (Runguphan and Keasling, 2014). Previous work has shown that a $S N F 2$ delete strain with over-expression of $D G A 1$ or a $D G A 1$ variant lacking the N-terminal 29 amino acids could accumulate lipids up to $45 \%$ and $50 \% \mathrm{dcw}$ on $5 \%$ and $10 \%$ glucose, respectively (Kamisaka et al., 2007; Kamisaka et al., 2013). The gene SNF2 codes for an ATPase subunit of the SWI-SNF chromatin remodeling complex (Peterson and Tamkun, 1995). Another engineering effort in S. cerevisiae showed a $63 \%$ increase in intracellular lipids by over-expression of native GPD1 and ACC1 from Lipomyces starkeyi (Wang et al., 2016).

Here we report the discovery of an oleaginous strain of S. cerevisiae which, through further genetic engineering, now utilizes the lignocellulosic biomass derived xylose monomer to produce ethanol concurrently with increased lipid accumulation through a $S N F 1$ knock-out and $D G A$ over-expression. In this manuscript, we demonstrate high lipid accumulation in a $S N F 1$ knock-out $S$. cerevisiae strain which is also capable of utilizing xylose presenting an opportunity to produce a lipid feedstock from cellulosic biomass.

\section{Materials and Methods}

\subsection{Yeast strains, media, and seed culture growth conditions}

The S. cerevisiae strains used in this study are listed in Table 1 . Yeast strains were grown in YPD (Difco) at $30^{\circ} \mathrm{C}$ with shaking at $225 \mathrm{rpm}$ for seed culture generation and strain maintenance. For lipid accumulation, yeast strains were grown in yeast nitrogen base (YNB) (Sigma Y-0626) containing 5\% glucose and $5 \mathrm{mM} \mathrm{NH}_{4}{ }^{+}$. For shake flask studies, cultures were grown at $30^{\circ} \mathrm{C}$ with shaking at $225 \mathrm{rpm}$. Seed cultures were grown in $100 \mathrm{~mL}$ YPD in a $500 \mathrm{~mL}$ baffled shake flask overnight. For lipid accumulation, yeasts were grown in $300 \mathrm{~mL}$ of media in a $1 \mathrm{~L}$ baffled flask in duplicate inoculated with washed cells from an overnight YPD seed culture to an initial $\mathrm{OD}_{600}$ of 1 . Cultures were incubated at $30^{\circ} \mathrm{C}$ at $225 \mathrm{rpm}$. At each time point, $45 \mathrm{~mL}$ of culture was collected. From this sample, $5 \mathrm{~mL}$ were removed for $\mathrm{OD}_{600}$, HPLC, and YSI analysis and the remaining $40 \mathrm{~mL}$ of culture was pelleted and frozen at $-80^{\circ} \mathrm{C}$ for in-situ FAME analysis.

Table 1.

S. cerevisiae strains used in this study.

\begin{tabular}{lll}
\hline Yeast strain & Relevant genotype & Reference \\
\hline AGL & WT & Angel Yeast Co. Ltd. \\
D5A & WT & Bailey et al. (1982) \\
Fali & WT & Broin, Inc. \\
PE-2 & WT & Basso et al. (2008) \\
BFY692 & D5A; GAL80 $:: P S X Y L 1-P s X Y L 2-S c X K S$ & This study \\
BFY709 & BFY692; Snf1 & This study \\
BFY742 & BFY709; ScDGA1 & This study \\
BFY746 & BFY709; ScDga1 $\triangle$ Np & This study \\
BFY748 & BFY709; LsDGA1 & This study \\
\hline
\end{tabular}

\subsection{Genetic methods}

The xylose reductase (XYL1), xylitol dehydrogenase (XYL2), and xylulose kinase $(X K S)$ expression construct with 500 bp of GAL80 targeting flanking sequences was synthesized (DNA2.0). The PGK1 promoter and GAL10 terminator were used to drive $X Y L 1$ expression. The $T D H 3$ promoter and GAL2 terminator were used to drive $X Y L 2$ expression and the $P G I 1$ promoter and $P D C 1$ terminator were used to drive $X K S 1$ expression. The construct was isolated from the vector with the restriction enzyme NotI and $1 \mu \mathrm{g}$ of the gel-purified expression construct DNA was transformed into S. cerevisiae D5A for integration at GAL80.

The SNF1 knockout was generated in two steps. Two SNF1-targeting constructs were built either having G418 or hygromycin B as the selection markers. For integrative targeting, 690 and $400 \mathrm{bp}$ of sequence upstream and downstream of the $S N F 1$ locus, respectively, were added to either end of the constructs. In both constructs, the selection marker was driven by the $P G K 1$ promoter and the GAL2 terminator.

The various DGA genes were PCR amplified (ScDGA1 (YOR245C)), or synthesized as a geneblock (Integrated DNA technologies Inc.) (LsDGA1 (synthesized with 3 introns removed, gm1.6201_g)). The ScDGA1-truncated (Kamisaka et al., 2013) was constructed using PCR by amplifying the entire wild type $\mathrm{Sc} D G A 1$ vector using primers starting on either side of the region to be eliminated and having 15-bp overlaps with each other to utilize Gibson assembly (New England Biolabs Inc., cat no. E2611S) to close the circle. All DGA genes were expressed by the TDH3 promoter and GAL2 terminator.

Transformation of linear or plasmid DNA into S. cerevisiae D5A was performed using a DMSO-enhanced lithium-acetate protocol (Hill et al., 1991) with the following modifications. Cells were initially washed in water. Six-hundred $\mu \mathrm{L}$ of PEG4000 solution was added and just prior to heat shocking, $70 \mu \mathrm{L}$ DMSO was added. Cells were heat-shocked for 15 $\min$ at $42^{\circ} \mathrm{C}$ and the last wash step was eliminated. Cells were re-suspended in $10 \mathrm{mM}$ Tris-EDTA and plated on appropriate selective plates. Electrotransformation of E. coli Dh5 $\alpha$ was performed as described (Invitrogen 11319-019) and plated on LB plates containing $100 \mu \mathrm{g} / \mathrm{mL}$ ampicillin. E. coli plasmid DNA was isolated using a plasmid spin miniprep kit (Qiagen Inc., cat no. 27106).

\subsection{Analytical methods}

Concentrations of sugars and ethanol were measured using a high performance liquid chromatograph (HPLC) equipped with an HP refractive index detectors (Agilent Technologies, Palo Alto, CA). A Bio-Rad HPX- 
$87 \mathrm{H}$ organic acids column and $\mathrm{H}+$ guard column (BioRad Laboratories, Hercules, CA) operated at $55{ }^{\circ} \mathrm{C}$ were used. The eluent was $0.01 \mathrm{~N} \mathrm{H}_{2} \mathrm{SO}_{4}$ at a flow rate of $0.6 \mathrm{~mL} \mathrm{~min}^{-1}$. Samples and standards were filtered through 0.45 $\mathrm{mm}$ nylon membrane syringe filters (Pall Corp., East Hills, NY) prior to injection onto the column.

Intracellular lipid accumulation was measured as fatty acid methyl esters (FAMEs) after in situ transesterification of the endogenous lipids to FAME followed by GC analysis as follows: 7 to $10 \mathrm{mg}$ of lyophilized microbial biomass (dried overnight at $40^{\circ} \mathrm{C}$ under vacuum) was homogenized with 0.2 $\mathrm{mL}$ of chloroform:methanol $(2: 1 \mathrm{v} / \mathrm{v})$, and the resulting solubilized lipids were transesterified in situ with $0.3 \mathrm{~mL}$ of $\mathrm{HCl}$ :methanol $\left(5 \%\right.$, v/v) for $1 \mathrm{~h}$ at $85^{\circ} \mathrm{C}$ in the presence of tridecanoic acid (C13) methyl ester as an internal standard. FAMEs were extracted with $1 \mathrm{~mL}$ of hexane at room temperature for $1 \mathrm{~h}$ and analyzed by gas chromatography:flame ionization detection (GC:FID) on an Agilent (Santa Clara, CA, USA) $6890 \mathrm{~N}$ with a DB-WAX column with dimensions $30 \mathrm{~m} \times 0.25 \mathrm{~mm}$ i.d. and $0.25 \mu \mathrm{m}$ film thickness. Individual fatty acids were identified by mass spectrometry for the location of the unsaturation of the fatty acid positional isomers. The FAMEs were quantified based on a 37FAME calibration mixture (Supelco, certified reference material, CRM47885, Sigma-Aldrich, St. Louis, MO, USA) after normalizing for the internal standard. The sum of the individual fatty acids was calculated and expressed as weight $\%$ of dry biomass (Laurens et al., 2012).

\subsection{Imaging}

Images were acquired by staining a $100 \mathrm{uL}$ cell suspension in water with 1 $\mathrm{uL}$ of Nile Red dissolved in acetone at $250 \mathrm{ug} / \mathrm{mL}$. Cells and stain were allowed to incubate for $5 \mathrm{~min}$ prior to imaging. Images were collected on a Nikon epifluorescent microscope with $100 \times$ objective. Cells were illuminated by a mercury lamp with $480 / 40 \mathrm{~nm}$ band pass excitation and $505 \mathrm{~nm}$ long pass emission filter sets.

\section{Results and Discussion}

\subsection{Identification of naturally oleaginous S. cerevisiae}

Four strains of $S$. cerevisiae previously used in industrial scale ethanol fermentations were characterized for lipid accumulation (Fig. 1A). Unique among these four strains was D5A which accumulated 20\% FAME on a dcw basis in a nitrogen limited culture (5mM NH 4 initial concentration) compared to less than $15 \%$ dcw FAME for the other 3 strains. The rates of glucose utilization and ethanol production were similar among the four strains except for PE-2 which produced significantly more ethanol (Fig. 1B). PE-2 is known to give higher ethanol titers (Basso et al., 2008). Ethanol begins to disappear after $48 \mathrm{~h}$ due to the aerobic nature of the culture. The strains PE-2 and D5A also had lower final optical densities due to carbon being funneled to ethanol or lipids, respectively, rather than cell growth.
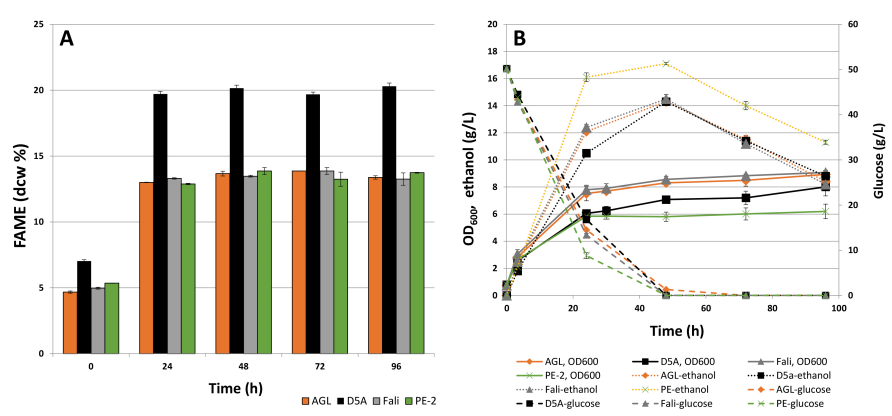

Fig.1. Comparison of industrial strains of S. cerevisiae. A: Lipid accumulation (\% dew FAME). B: Optical density, solid lines; glucose, dashed lines; and ethanol, dotted lines.

\subsection{Engineering of $S$. cerevisiae for xylose utilization and increased lipid accumulation}

With the identification of D5A as naturally oleaginous, we decided to further develop the strain for biofuels production by focusing on the strain's pentose sugar utilization. Typical $S$. cerevisiae is not natively competent to utilize xylose, thus we engineered D5A by integration of a three-gene xylose utilization expression cassette. An expression construct harboring XYL1 and XYL2 from Pichia stipitis and XKS1 from S. cerevisiae was integrated at the chromosomal GAL80 locus completely replacing the GAL80 open reading frame to create strain BFY692. Complete replacement of $G A L 80$ was confirmed by PCR. Insertion at the GAL80 locus served to remove Gal80p repression of the galactose catabolic genes when glucose is present providing active expression of the Gal2p transporter. The Gal2p transporter has been well characterized as a major transporter of the pentose sugars xylose and arabinose, as well as glucose and galactose (Hamacher et al., 2002; Sedlak and Ho, 2004; Salheimo et al., 2007; Knoshaug et al., 2015). Engineering of the S. cerevisiae D5A strain for xylose utilization has been previously described (Hector et al., 2011), however, our strategy differed in that we targeted integration of our three-gene expression cassette to knock-out a negative regulator of a key pentose transporter (Gal2p) to ensure effective expression of this transporter in the presence of glucose. We then further engineered BFY692 for increased lipid accumulation by deleting the global regulator $S N F 1$ as it has previously been shown to play an important role in lipid accumulation in both $S$. cerevisiae and $Y$. lipolytica (Chumnanpuen et al., 2012; Seip et al., 2013). This required a two-step process as D5A is a diploid yeast (Bailey et al., 1982). We first knocked out one copy using a $S N F 1$ targeted G418 resistance integration cassette, then the other copy using a similarly targeted Hygromycin B resistance integration cassette. Integration at one locus, then the other was confirmed by PCR. When grown for lipid accumulation in a nitrogenlimited culture, 34\% dcw FAME accumulated in the double SNF1 knock out strain BFY709 compared to 23\% in the BFY692 parent (Fig. 2A). It was interesting to note that to realize an increase in lipid accumulation, both copies of SNF1 had to be knocked out indicating that SNF1 is a dominant gene, as there was no intermediate response with only one copy of SNF1. In these conditions, one copy of SNF1 was adequate to perform its regulatory duties blocking increased lipid accumulation. In addition, though the double $S N F 1$ knock-out strain accumulates considerably more lipids than the parent, nearly the same amount of ethanol is produced by the end of the fermentation (Fig. 2B)
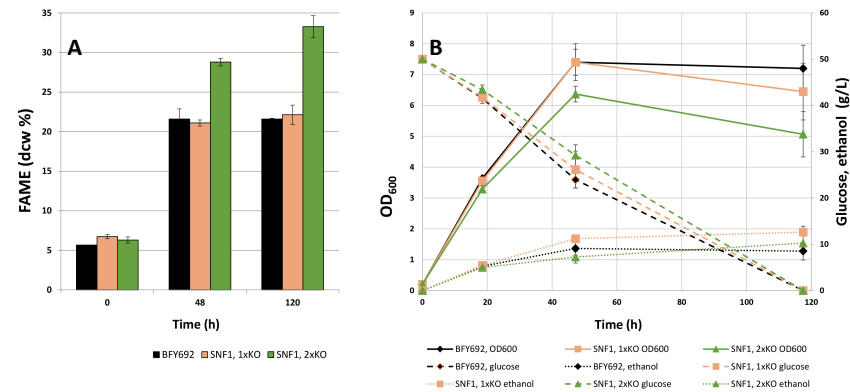

Fig.2. Lipid, optical density, and glucose utilization in aerobic shake flasks. S. cerevisiae strains: A: Lipid accumulation. B: Optical density, solid lines; glucose utilization, dashed lines; ethanol production, dotted lines.

Glucose utilization and growth rate between the parent strain and the single or double Snf1 ${ }^{-}$strains were similar with the double Snf1 ${ }^{-}$strain reaching a slightly lower $\mathrm{OD}_{600}$. The lower cell density could possibly be explained by the funneling of more of the available carbon into lipid accumulation rather than cell growth in the double knock-out strain. It is 
important to note that even though these were aerobic shake flasks, ethanol was produced and later metabolized to some degree, showing that ethanol and lipids can be produced simultaneously. With the decrease in glycogen synthesis and $\beta$-oxidation and the increase in the acetyl-CoA pool due to the loss of Snflp regulation of these pathways, a plausible mechanism for the increase in lipid accumulation is that carbon flux now funnels into the de-novo lipid synthesis pathway while blocking lipids from oxidation.
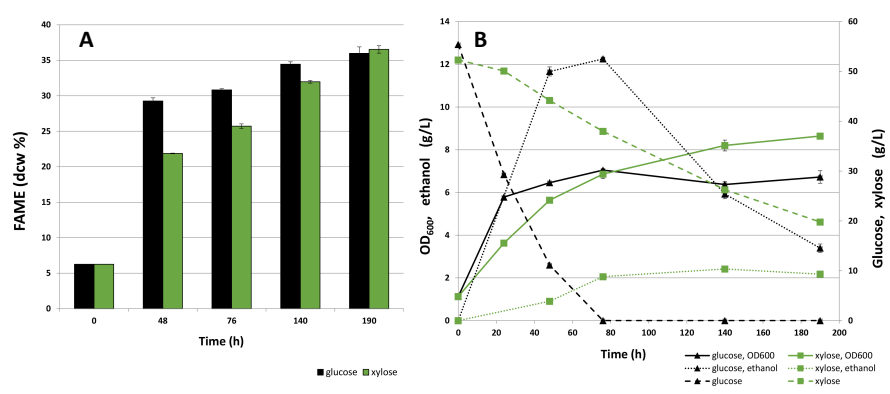

Fig.3. Lipid and ethanol production, growth, and sugar utilization by S. cerevisiae BFY709 in aerobic shake flasks. A: Lipid accumulation. B: Optical density, solid lines; sugar utilization, dashed lines; and ethanol production, dotted lines.

Strain BFY709 accumulated up to $36 \%$ FAME dcw from xylose as the sole carbon source (Fig. 3A). The accumulation of lipids from xylose took longer than when grown on glucose while ethanol production from xylose was much lower (Fig. 3B). Up to $0.5 \mathrm{~g} / \mathrm{L}$ xylitol were also produced when grown on xylose. Cell density was slightly higher when grown on xylose while the consumption of xylose was considerably slower than the consumption of glucose with nearly half of the xylose still present after $190 \mathrm{~h}$ of incubation (Fig. 3B). This data suggests that carbon liberated from xylose is first shunted to lipid synthesis, with ethanol production being secondary. Using the introduced pathway, xylose is converted to D-xylulose-5-phosphate which then directly enters the pentose phosphate pathway (PPP). Glyceraldehyde-3phosphate is readily isomerized to DHAP which can then enter the lipid pathway by conversion to glycerol-3-phosphate. Previous work has shown that $S$. cerevisiae has insufficient levels of TAL1 to efficiently ferment xylose (Walfridsson et al., 1995; Hasunuma et al., 2011), causing a reduced capacity of flux through the PPP and a build-up of sedoheptulose-7-phosphate (Kotter and Ciriacy, 1993). Reduced oxidative PPP flux improves ethanol yield (Jeppsson et al., 2002) suggesting that having a slower carbon flux through the PPP allows time for the lipid production pathway enzymes to siphon off carbon and shunt it to lipids rather than ethanol. However, previous research has also shown that sufficient flux through the PPP is needed for NADPH regeneration for increased lipid production in Y. lipolytica (Wasylenko et al., 2015). To further complicate this issue, it has also been shown that the non-oxidative PPP controls the rate of xylulose fermentation but not xylose fermentation (Johansson and Hahn-Hagerdal, 2002) suggesting that upstream barriers to xylose fermentation are in transport, xylose reductase, xylitol dehydrogenase, or xylulokinase. Clearly xylose (carbon) flux through the PPP is slow in our engineered strain and for unknown reasons leads to high lipid content (as high as glucose in the same time frame) at the expense of ethanol production even when the strain is unable to efficiently ferment all of the available xylose. The slower rate and inability to effectively utilize all of the xylose is likely due to exhaustion of the available nitrogen in the media as the fermentation was conducted in minimal media having an initial $\mathrm{NH}_{4}{ }^{+}$concentration of only 5 $\mathrm{mM}$. This low initial amount of $\mathrm{NH}_{4}{ }^{+}$is typically reduced to undetectable levels within $6 \mathrm{~h}$ after inoculation (data not shown). In addition, ethanol production appears to cease while internal lipids continue to increase after $80 \mathrm{~h}$. However, in aerobic flasks, it is possible that ethanol metabolism is occurring and thus reducing accumulation in the media though this is unlikely as xylose continues to decrease linearly. Given the complexities of possible carbon flux scenarios for glucose and xylose above, studies are underway to elucidate the basis for oleaginicity in this naturally oleaginous $S$. cerevisiae strain D5A. To visualize the internal lipids, Nile Red was used to stain lipid bodies within the cells after $76 \mathrm{~h}$ of growth on glucose or xylose (Fig. 4). Side-by-side images display two different focal planes within the same cells showing that multiple layers of lipid bodies exist throughout the cells but there were no large differences in lipid body size, number, or distribution observed between cells grown on glucose or xylose.

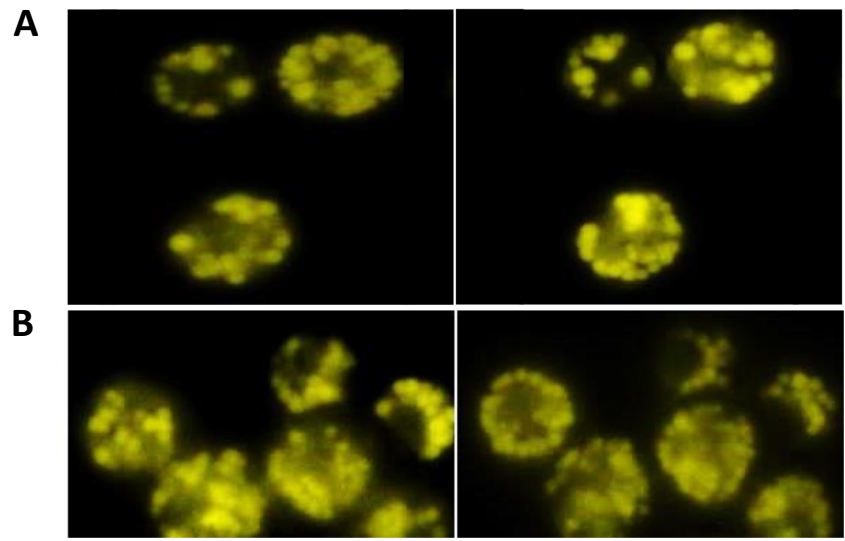

Fig.4. Nile Red stained accumulated lipids in cells of S. cerevisiae BFY709. A: glucose or B: xylose. The two panels show the same cells with different focal planes illustrating the different internal layering of stained lipid bodies.

To further increase lipids, we over-expressed DGA (BFY742, ScDGA1), a truncated version (BFY746, ScDga1 $\triangle \mathrm{Np}$, (Kamisaka et al., 2013)), and a DGA from L. starkeyi (BFY748, LsDGA1) in strain BFY709 (Fig. 5). We observed up to $50 \%$ dcw FAME when over-expressing the native $D G A 1$ or the truncated version and $45 \%$ dcw FAME with $L$. starkeyi DGA1. High levels of lipids were also seen at an increased temperature of $37^{\circ} \mathrm{C}$ though slightly lower than lipid levels at $30^{\circ} \mathrm{C}$. The optical densities $\left(\mathrm{OD}_{600}<8\right)$, glucose utilization, and ethanol production $(<14 \mathrm{~g} / \mathrm{L})$ for these strains were similar to those seen in previous experiments for D5A strains (data not shown).

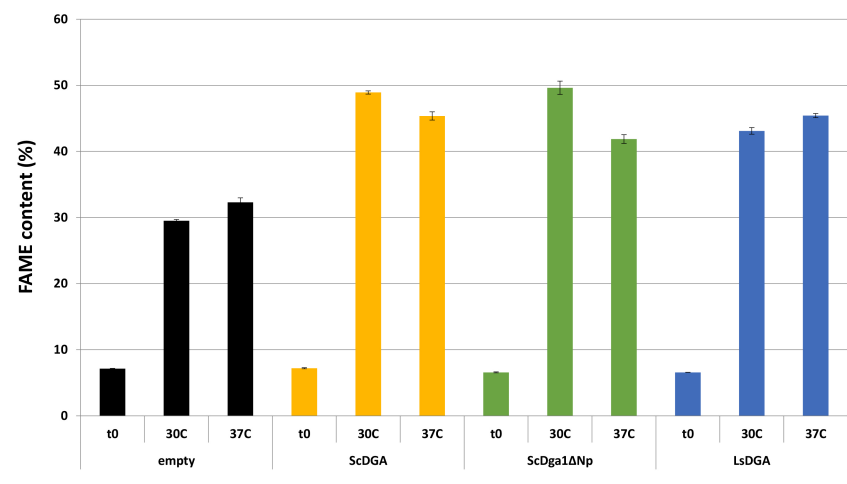

Fig.5. Maximum lipid content in D5A expressing different $D G A 1$ genes in seed cultures at t0 and 30 or $37^{\circ} \mathrm{C}$

\subsection{Fatty acid distribution in S. cerevisiae}

The distribution of fatty acids changed over the course of the fermentations. In all, 21 different fatty acids species were found yet only 5 
fatty acid species, C16:0 (palmitic), C16:1n7 (palmitoleic), C18:0 (stearic), C18:1n7 (vaccenic), and C18:1n9 (oleic) made up $>94 \%$ of the total fatty acids (Fig. 6). Over the course of the fermentations, when glucose was the sole carbon source in a defined media, $\mathrm{C} 16: 1 \mathrm{n} 7$ decreased, $\mathrm{C} 18: 0$ and $\mathrm{C} 18: 1 \mathrm{n} 9$ increased, and $\mathrm{C} 16: 0$ and $\mathrm{C} 18: 1 \mathrm{n} 7$ acids did not change appreciably. In contrast, when xylose was the sole carbon source in defined media, C16:1n7 and $\mathrm{C} 18: 1 \mathrm{n} 9$ decreased, $\mathrm{C} 16: 0$ and $\mathrm{C} 18: 0$ increased, while $\mathrm{C} 18: 1 \mathrm{n} 7$ remained unchanged signaling a small shift from unsaturated to saturated (Fig. 6). Our fatty acid speciation data agrees with previously reported values of $\mathrm{C} 16: 1$ and C18:1 being the main species present (>72\%), with C16:0 and C18:0 making up the bulk of the remainder (Kamisaka et al., 2013). The fatty acid speciation was different from that of oleaginous yeast of the Lipomycetaceae family in that $\mathrm{C} 16: 0$ was typically between $15-50 \%$ whereas $\mathrm{C} 16: 1$ was typically $<10 \%$ for the majority of the strains with the exception of some species of Myxozyma (Van Rensburg et al., 1995). Recent fatty acid engineering efforts have shown the potential to change levels of different species of the fatty acids present in cells making possible designer ratios of lipids and fatty acid chain lengths (Tang et al., 2013) as well as efforts to produce free fatty acids, fatty alcohols, and fatty acid ethyl esters (Runguphan and Keasling, 2014). Clearly with the range of facile engineering tools and wealth of information available for $S$. cerevisiae, this yeast is being actively developed and has a huge potential for producing lipid-based fuels, designer lipids, fatty acids, and other hydrocarbonbased chemicals.

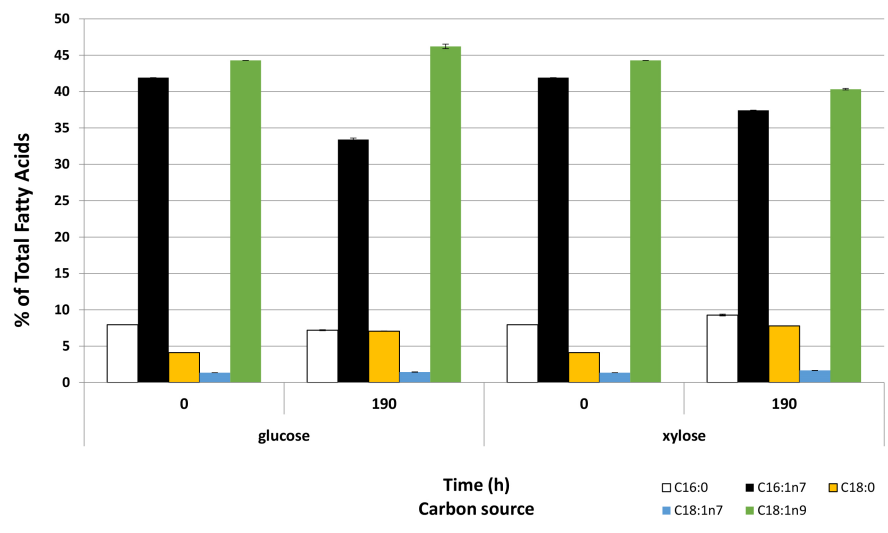

Fig.6. Changes in fatty acid speciation over time in glucose or xylose.

\section{Conclusions}

In conclusion, we have developed an oleaginous $S$. cerevisiae strain that concurrently produces high titers of ethanol with up to 50\% lipids from either glucose or xylose. As interest in renewable biofuels continues to increase, genetic engineering efforts will continue in $S$. cerevisiae to develop nextgeneration biocatalysts with expanded product ranges from a variety of feedstock sugars.

\section{Acknowledgments}

This work was supported by the U.S. Department of Energy under Contract No. DE-AC36-08GO28308 with the National Renewable Energy Laboratory. Funding was provided by U.S. DOE Office of Energy Efficiency and Renewable Energy, Bioenergy Technologies Office. The U.S. Government retains and the publisher, by accepting the article for publication, acknowledges that the U.S. Government retains a nonexclusive, paid-up, irrevocable, worldwide license to publish or reproduce the published form of this work, or allow others to do so, for U.S. Government purposes.

\section{References}

[1] Ageitos, J.M., Vallejo, J.A., Veiga-Crespo, P., Villa, T.G., 2011. Oily yeasts as oleaginous cell factories. Appl. Microbiol. Biotechnol. 90(4), $1219-1227$
[2] Bailey, R.B., Benitez, T., Woodward, A., 1982. Saccharomyces cerevisiae mutants resistant to catabolite repression: use in cheese whey hydrolysate fermentation. Appl. Environ. Microbiol. 44(3), 631-639.

[3] Basso, L.C., de Amorim, H.V., de Oliveira, A.J., Lopes, M.L., 2008 Yeast selection for fuel ethanol production in Brazil. FEMS Yeas Res. 8(7), 1155-1163.

[4] Bera, A.K., Sedlak, M., Khan, A., Ho, N.W., 2010. Establishment of L-arabinose fermentation in glucose/xylose co-fermenting recombinant Saccharomyces cerevisiae 424A (LNH-ST) by genetic engineering. Appl. Biochem. Biotechnol. 87(5), 1803-1811.

[5] Bettiga, M., Bengtsson, O., Hahn-Hagerdal, B., Gorwa-Grauslund, M.F., 2009. Arabinose and xylose fermentation by recombinant Saccharomyces cerevisiae expressing a fungal pentose utilization pathway. Microb. Cell Fact. 8(1), 40.

[6] Brat, D., Boles, E., Wiedemann, B., 2009. Functional expression of a bacterial xylose isomerase in Sacharomyces cerevisiae. Appl. Environ. Microbiol. 75(8), 2304-2311.

[7] Chumnanpuen, P., Zhang, J., Nookaew, I., Nielsen, J. 2012 Integrated analysis of transcriptome and lipid profiling reveals the coinfluences of inositol-choline and Snf1 in controlling lipid biosynthesis in yeast. Mol. Genet. Genomics. 287(7), 541-554.

[8] de Figueiredo Vilela, L., de Mello, V.M., Reis, V.C.B., da Silva Bon, E.P., Torres, F.A.G., Neves, B.C., Eleutherio, E.C.A., 2013 Functional expression of Burkholderia cenocepacia xylose isomerase in yeast increases ethanol production from a glucose-xylose blend. Bioresour. Technol. 128, 792-796.

[9] Geddes, C.C., Nieves, I.U., Ingram, L.O., 2011. Advances in ethanol production. Curr. Opin. Biotechnol. 22(3), 312-319.

[10] Greer, M.S., Truksa, M., Deng, W., Lung, S.C., Chen, G., Weselake, R.J., 2015. Engineering increased triacylglycerol accumulation in Saccharomyces cerevisiae using a modified type 1 plant diacylglycerol acyltransferase. Appl. Microbiol. Biotechnol. 99(5), 2243-2253.

[11] Hamacher, T., Becker, J., Gardonyi, M., Hahn-Hagerdal, B., Boles, E., 2002. Characterization of the xylose-transporting properties of yeast hexose transporters and their influence on xylose utilization. Microbiology. 148(9), 2783-2788

[12] Hardie, D.G., Carling, D., Carlson, M., 1998. The AMPactivated/SNF1 protein kinase subfamily: metabolic sensors of the eukaryotic cell?. Annu. Rev. Biochem. 67, 821-855.

[13] Hasunuma, T., Sandra, T., Yamada, R., Yoshimura, K., Ishii, J., Kondo, A., 2011. Metabolic pathway engineering based on metabolomics confers acetic and formic acid tolerance to a recombinant xylose-fermenting strain of Saccharomyces cerevisiae Microb. Cell Fact. 10(1), 2.

[14] Hector, R.E., Dien, B.S., Cotta, M.A., Qureshi, N., 2011. Engineering industrial Saccharomyces cerevisiae strains for xylose fermentation and comparison for switchgrass conversion. J. Ind. Microbiol. Biotechnol. 38(9), 1193-1202.

[15] Hill, J., Donald, K.A., Griffiths, D.E., Donald, G., 1991. DMSOenhanced whole cell yeast transformation. Nucleic Acids Res. 19(20), 5791

[16] Jeppsson, M., Johansson, B., Hahn-Hagerdal, B., Gorwa-Grauslund, M.F. 2002. Reduced oxidative pentose phosphate pathway flux in recombinant xylose-utilizing Saccharomyces cerevisiae strain improves the ethanol yield from xylose. Appl. Environ. Microbiol. 68(4), 1604-1609

[17] Johansson, B., Hahn-Hagerdal, B., 2002. The non-oxidative pentose phosphate pathway controls the fermentation rate of xylulose but not of xylose in Saccharomyces cerevisiae TMB3001. FEMS Yeast Res. 2(3), 277-282.

[18] Kamisaka, Y., Kimura, K., Uemura, H., Yamaoka, M., 2013. Overexpression of the active diacylglycerol acyltransferase variant transforms Saccharomyces cerevisiae into an oleaginous yeast. Appl. Microbiol. Biotechnol. 97(16), 7345-7355.

[19] Kamisaka, Y., Tomita, N., Kimura, K., Kainou, K., Uemura, H., 2007. DGA1 (diacylglycerol acyltransferase gene) overexpression and leucine biosynthesis significantly increase lipid accumulation in 
the $\Delta$ snf2 disruptant of Saccharomyces cerevisiae. Biochem. J. 408(1), 61-68.

[20] Karhumaa, K., Wiedemann, B., Hahn-Hagerdal, B., Boles, E., GorwaGrauslund, M.F., 2006. Co-utilization of L-arabinose and D-xylose by laboratory and industrial Saccharomyces cerevisiae strains. Microb. Cell Fact. 5(1), 8.

[21] Knoshaug, E.P., Vidgren, V., Magalhaes, F., Jarvis, E.E., Franden, M.A., Zhang, M., Singh, A., 2015. Novel transporters from Kluyveromyces marxianus and Pichia guilliermondii expressed in Saccharomyces cerevisiae enable growth on L-arabinose and D-xylose. Yeast. 32(10), 615-628.

[22] Knoshaug, E.P., Zhang, M., 2009. Butanol tolerance in a selection of microorganisms. Appl. Biochem. Biotechnol. 153(1-3), 13-20.

[23] Kotter, P., Amore, R., Hollenberg, C.P., Ciriacy, M., 1990. Isolation and characterization of the Pichia stipitis xylitol dehydrogenase gene, $\mathrm{XYL}_{2}$, and construction of a xylose-utilizing Saccharomyces cerevisiae transformant. Curr. Genet. 18(6), 493-500.

[24] Kotter, P., Ciriacy, M., 1993. Xylose fermentation by Saccharomyces cerevisiae. Appl. Microbiol Biotechnol. 38(3), 776-783.

[25] Laurens, L.M., Quinn, M., Van Wychen, S., Templeton, D.W., Wolfrum, E.J., 2012. Accurate and reliable quantification of total microalgal fuel potential as fatty acid methyl esters by in situ transesterification. Anal. Bioanal. Chem. 403(1), 167-178.

[26] Mayer, F.V., Heath, R., Underwood, E., Sanders, M.J., Carmena, D., McCartney, R.R., Leiper, F.C., Xiao, B., Jing, C., Walker, P.A., Haire, L.F., Ogrodowica, R., Martin, S.R., Schmidt, M.C., Gamblin, S.J., Carling, D., 2011., ADP regulates SNF1, the Saccharomyces cerevisiae homolog of AMP-activated protein kinase. Cell Metab. 14(5), 707-714.

[27] Meng, X., Yang, J., Xu, X., Zhang, L., Nie, Q., Xian, M., 2009. Biodiesel production from oleaginous microorganisms. Renewable Energy. 34(1), $1-5$

[28] Mitchelhill, K.I., Stapleton, D., Gao, G., House, C., Michell, B., Katsis, F., Witters, L.A., Kemp, B.E., 1994. Mammalian AMP-activated protein kinase shares structural and functional homolgy with the catalytic domain of yeast Snf1 protein kinase. J. Biol. Chem. 269(4), 2361-2364.

[29] Peterson, C.L., Tamkun, J.W., 1995. The SWI-SNF complex: a chromatin remodeling machine?. Trends Biochem. Sci. 20(4), 143-146.

[30] Ranatunga, T.D., Jervis, J., Helm, R.F., McMillan, J.D., Hatzis, C., 1997. Toxicity of hardwood extractives toward Saccharomyces cerevisiae glucose fermentation. Biotechnol. Lett. 19(11), 1125-1127.

[31] Ratledge, C., Wynn, J.P., 2002. The biochemistry and molecular biology of lipid accumulation in oleaginous microorganisms. Adv. Appl. Microbiol. 51, 1-52.

[32] Runguphan, W., Keasling, J.D., 2014. Metabolic engineering of Saccharomyces cerevisiae for production of fatty acid-derived biofuels and chemicals. Metab. Eng. 21, 103-113.

[33] Salheimo, A., Rauta, J., Stasyk, V., Sibirny, A.A., Penttila, M., Ruohonen, L., 2007. Xylose transport studies with xylose-utilizing Saccharomyces cerevisiae strains expressing heterologous and homologous permeases. Appl. Microbiol. Biotechnol. 74(5), 1041-1052.
[34] Sedlak, M., Ho, N.W., 2004. Characterization of the effectiveness of hexose transporters for transporting xylose during glucose and xylose co-fermentation by a recombinant Saccharomyces yeast. Yeast. 21(8), 671-684.

[35] Seip, J., Jackson, R., He, H., Zhu, Q., Hong, S.P., 2013. Snf1 is a regulator of a lipid accumulation in Yarrowia lipolytica. Appl. Environ. Microbiol. 79(23), 7360-7370.

[36] Tang, X., Feng, H., Chen, W.N., 2013. Metabolic engineering for enhanced fatty acids synthesis in Saccharomyces cerevisiae. Metab. Eng. 16, 95-102

[37] Thompson-Jaeger, S., Francois, J., Gaughran, J.P., Tatchell, K., 1991. Deletion of SNF1 affects the nutrient response of yeast and resembles mutations which activate the adenylate cyclase pathway. Genetics. 129(3), 697-706

[38] Turcotte, G., Kosaric, N., 1989. Lipid biosynthesis in oleaginous yeasts. Adv. Biochem. Eng. Biotechnol. 40, 73-92.

[39] Usaite, R., Jewett, M.C., Oliveira, A.P., Yates, J.R., Olsson, L. Nielsen, J., 2009. Reconstruction of the yeast Snf1 kinase regulatory network reveals its role as a global energy regulator. Mol. Syst. Biol. 5(1), 319.

[40] Van Rensburg, E.L.J., Kock, J.L.F., Coetzee, D.J., Botha, A., Botes, P.J., 1995. Lipid composition and DNA band patterns in the yeast family Lipomycetaceae. Syst. Appl. Micriobiol. 18(3), 410-424.

[41] Walfridsson, M., Hallborn, J., Penttila, M.E.R.J.A., Keranen, S.I.R.K.K.A., Hahn-Hagerdal, B., 1995. Xylose-metabolizing Saccharomyces cerevisiae strains overexpressing the TKL1 and TAL1 genes encoding the pentose phosphate pathway enzymes transketolase and transaldolase. Appl. Environ. Microbiol. 61(12), 4184-4190.

[42] Wang, J., Xu, R., Wang, R., Haque, M.E., Liu, A., 2016. Overexpression of ACC gene from oleaginous yeast Lipomyces starkeyi enhanced the lipid accumulation in Saccharomyces cerevisiae with increased levels of glycerol 3-phosphate substrates. Biosci. Biotechnol. Biochem. 80(6), 1214-1222.

[43] Wasylenko, T.M., Ahn, W.S., Stephanopoulos, G., 2015. The oxidative pentose phosphate pathway is the primary source of NADPH for lipid overproduction from glucose in Yarrowia lipolytica. Metab. Eng. 30, 27-39.

[44] Woods, A., Munday, M.R., Scott, J., Yang, X., Carlson, M., Carling, D., 1994. Yeast SNF1 is functionally related to mammalian AMPactivated protein kinase and regulates acetyl-CoA carboxylase in vivo. J. Biol. Chem. 269(30), 19509-19515.

[45] Yu, K.O., Jung, J., Ramzi, A.B., Choe, S.H., Kim, S.W., Park, C., Han, S.O., 2013. Development of a Saccharomyces cerevisiae strain for increasing the accumulation of triacylglycerol as a microbial oil feedstock for biodiesel production using glycerol as a substrate. Biotechnol. Bioeng. 110(10), 343-347.

[46] Zaman, S., Lippman, S.I., Zhao, X., Broach, J.R., 2008. How Saccharomyces responds to nutrients. Annu. Rev. Genet. 42, 27-81. 